recall. Journal of Verbal Learning and Verbal Behavior, 1972, 11, 717-726.

Dooling, D. J., \& Lachman, R. Effects of comprehenson on retention of prose. Journal of Experimental Psychology, retention of prose.

Heinen, J. R. K., Kulhavy, R. W., \& Dyer, J. W. Context and the meaning of words in paragraphs. Paper presented at the Annual Convention of the American Psychological
Association, New Orleans, August 1974.

Kulhavy $R$ W. Effects of embedding orienting stimuli in a prose passage. Psychonomic Science, 1972, 28, 213-214.

Kulhavy, R. W., \& Heinen, J. R. K. Mnemonic transformations and verbal coding processes. Journal of Experimental Psychology, 1974, 102, 173-175.

(Received for publication August 13, 1974.)

\title{
On the recall of nonverbal experiences
}

\author{
JOHN G. SEAMON \\ Wesleyan University, Middletown, Connecticut 06457
}

\begin{abstract}
The inability to recall early childhood experiences had led some theorists to suggest that the development of a visual representational system precedes that of a verbal system. Events perceived prior to the onset of the verbal system are unable to be recalled subsequently through that system. The present experiment, however, has demonstrated that subjects could attach verbal labels to nonverbal stimuli which were experienced prior to the establishment of rules for labeling. This suggests that poor recall of early experiences may reflect true forgetting rather than information inaccessibility. Assimilation into a permanent memory structure may be a necessary conditon for long-term retention.
\end{abstract}

Why are we unable to recall many events from our early childhood especially events which occurred before the age of two or three? A number of theorists who have considered this issue have suggested that there may be separate modes of thought which develop differentially in man. The two modes may be conceptualized as visual and verbal representational systems with the temporal onset of the former preceding that of the latter (Bower, 1970; Bruner, 1966; Gazzaniga, 1972; Neisser, 1962). Gazzaniga (1972, p. 315) summarizes this position of information inaccessibility by stating that "It is possible that the brain can remember critical events which may later play a role in the control of behavior, but because the remembered events occurred prior to the clear establishment of the language system they cannot be subsequently recalled through this system."

The present experiment was performed to examine an issue raised by the above theorists, viz, could the subjects attach verbal labels to nonverbal stimuli which were experienced prior to the establishement of rules for labeling? The ability of subjects to attach labels to their earlier nonverbal experiences would imply that our poor recall of early events may not be explained as a problem of information inaccessibility.

\section{METHOD}

Subjects

Thirty-two male and female Wesleyan University students

This research was supported by a Wealey an Univernity Faculty Research Grant. Requests for reprints should be sent to John G. Seamon, Department of Psychology, Wesleyan University, Middletown, Connecticut 06457.
}

served as paid subjects in this experiment.

\section{Materials}

The stimuli consisted of a pool of 18 nonsense shapes and 18 nonsense verbal labels individually printed on $7.5 \times 12.8 \mathrm{~cm}$ white cards. The shapes were geometric line drawings which varied in number of sides from 8 to 16 with a mean of 10.43 and filled the entire card. They were constructed by using only straight lines and forming angles by using a table of random numbers. The shapes generated were different from each other and not similar to any known geometric shapes or to any two-dimensional representations of real-life objects.

The nonsense labels, printed on separate cards, were pronounceable CVC syllables with no obvious meaningful associates e.g., RUP, DAK. The labels were typed in capital letters in the center of each card.

\section{Procedure}

The subjects were assigned to one of four experimental groups on a random basis and run individually. The groups differed by the order of presentation of the shape sequence and paired-associates (P-A) task, and by the type of stimuli used in the final recognition task. In the first condition (Shapes, P-A, 1) a subset of eight shapes was randomly selected for each subject from the pool of 18 and individually presented to the subjects with each card shown for $15 \mathrm{sec}$. The subjects were told to simply observe each shape and that additional instructions would follow. After the last shape was presented, a P-A task was employed in which the subjects had to learn a CVC label to each of eight shapes. The shapes used in the P-A task consisted of four shapes randomly selected from the previously viewed eight, and four completely new shapes not seen before and randomly selected from those remaining in the original pool. The subjects were not informed that some of the shapes in the P-A task were from the preceding sequence. A standard P-A format was used 
with a stimulus shape shown alone for $10 \mathrm{sec}$ followed by the shape and its response label together for an additional $10 \mathrm{sec}$. The eight names were randomly selected from the label pool for each shape and each subject. A trial consisted of exposure to all eight stimulus and response pairings. The subjects were required to respond with the correct verbal label to each stimulus within the time that the stimulus was shown alone to be counted as correct. A learning criterion of two perfect successive trials was employed with the order of the stimuli randomized on each trial.

Following satisfactory completion of the P-A criterion, subjects in the first condition were told that some of the original shapes were used in the P-A task and that they had learned labels to them. The subjects were asked to recall as many of the labels of the originally presented shapes as possible. The subjects were told to guess if they were not sure, but that only four labels could be recalled in total. After the recall test, the subjects in the first group were presented with a recognition test of labels. The subjects were simultaneoulsy presented with 12 labels consisting of the four correct labels associated with the originally presented shapes in the P-A task, the remaining four labels from the P-A task associated with shapes not in the original presentation, and four completely new labels randomly selected from the remainder of the names in the pool. The array of 12 labels was randomized and the subjects were required to select four labels and encouraged to guess if unsure.

The second condition (Shapes, P-A, 2) was identical to the first in all respects except the final recognition test. Instead of employing label stimuli as the to-be-recognized items, shapes were used. On the recognition test, these subjects were presented with four shapes from the original presentation and the P-A task, the remaining four shapes from the original presentation, the remaining four shapes from the P-A task, and four completely new shapes randomly selected from the remainder of the pool. These 16 shapes were simultaneously presented in a randomly arranged array, and the subjects were required to select the eight shapes from the original presentation. Guessing was again encouraged if necessary.

The third and fourth conditons (P-A, Shapes, 3 \& P-A, Shapes 4) served as controls and were the counterparts of Conditions 1 and 2 excepting that the P-A task preceded the presentation of the critical shape sequence. The final recognition test for Condition 3 employed labels, while Condition 4 used shapes. The same randomization and selection procedures were used in all phases of the experiment for all groups. Instructions in each case were the same with only minor wording changes between conditions owing to the different sequencing of phases and different recognition stimuli.

\section{RESULTS}

The central focus of this paper is the possibility of subjects' attaching verbal labels to their earlier nonverbal experiences. This was examined by comparing subjects who viewed geometric shapes prior to learning their appropriate verbal labels with subjects who learned the shape-label associations prior to viewing the sequence of shapes. It was expected that the control subjects who had prior knowledge of the labeling associations should perform perfectly on the recall and recognition tests as the exposure to a shape whose name is known should be equivalent to an experience of both, while subjects who acquired the shape labels subsequent to viewing the critical sequence may perform at either the same high level or a lower level with comparisons to chance necessary to determine the extent of these subjects' ability to label their prior experiences.
Recall and recognition memory performance between groups was tested by one-tailed analyses to see if postexposure labeling (Groups 1 \& 2; shapes, P-A) is equivalent or poorer than preexposure labeling (Groups $3 \& 4$; P-A, shapes) and by comparisons of group performance to chance. Postexposure labeling should not be greater than preexposure labeling under any of the conditions employed. Since Groups 1 and 2, 3 and 4, were treated alike through the recall test, the recall performance of these pairs of groups was combined to yield a larger subject base. Recall performance for each group, as well as a combination, is presented in Table 1. It may readily be seen that mean recall for Groups 3 and 4 , individually and together, is higher than that of Groups 1 and 2. The difference between the combined group means is significant, $\mathrm{t}(30)=4.65, \mathrm{p}<.001$, indicating that subjects were less efficient at postexposure labeling than preexposure labeling. The subjects in Groups 3 and 4 who saw the shape sequence after they had learned verbal labels to some of those shapes were able to recall the names of those shapes near maximal level with their performance significantly greater than chance recall of two names, $t(15)=9.94$, $p<.001$. The subjects in Groups 1 and 2 who saw the shape sequence prior to the P-A task were less efficient than the subjects with the reverse order, but these subjects were apparently still able to attach labels to some of the earlier viewed shapes as their performance was also significantly greater than chance, $t(15)=3.56$, $p<.01$. These findings suggest that subjects in all groups were capable of labeling some of the stimuli in the shape sequence in order to recall by name the visual stimuli, and that pre- and postexposure labeling were quantitatively, rather than qualitatively, different with the former easier than the latter. These recall differences between groups may not be explained by assuming that (a) subjects in Groups 3 and 4 rehearsed the names more frequently than subjects in Groups 1 and 2 since they had the P-A task first, and (b) subjects in Groups 1 and 2 suffered mild interference by implicitly and incorrectly labeling the shapes upon viewing them prior to the P-A task. The differential rehearsal hypothesis may be rejected by the finding of essentially zero correlations between the number of correct P-A trials of an item and its subsequent probability of recall for Groups 1 and 2 (r $=.068)$ and 3 and $4(r=.104)$. Similarly, an interference effect for subjects in Group 1 and 2 was not found. A comparison of the trial of last error for shapes seen previously and those not seen previously in the P-A task did not reveal a difference between the acquisition of names and previous exposure to the stimuli, $t(15)=$ $.008, \mathrm{p}>.10$.

A comparison of name recognition between Groups 1 and 3 in Table 1 reveals the same trend with performance for Group 3 significantly better than that of Group $1, t(14)=1.88, p<.05$. This is not surprising, as subjects to a considerable extent repeated their recall 
Table 1

Recall and Recognition Memory for Labels and Shapes Under Conditions of Pre- and Postexposure Labeling of Shapes

\begin{tabular}{clccc}
\hline $\begin{array}{c}\text { Experimental } \\
\text { Condition }\end{array}$ & & $\begin{array}{c}\text { Label } \\
\text { Recall } \\
(\mathrm{Max}=4)\end{array}$ & $\begin{array}{c}\text { Memory Test } \\
\text { Label } \\
\text { Recognition } \\
(\mathrm{Max}=4)\end{array}$ & $\begin{array}{c}\text { Shape } \\
\text { Recog- } \\
\text { nition } \\
(\mathrm{Max}=8)\end{array}$ \\
\hline Shapes, P-A, 1 & M & 2.50 & 2.50 & \\
& SD & .53 & 1.20 & \\
Shapes, P-A, 2 & M & 2.63 & & 5.50 \\
& SD & .74 & & 1.20 \\
Combined 1 and 2 & M & 2.56 & & \\
P-A, Shapes, 3 & M & .63 & & \\
& SD & .74 & 3.38 & \\
P-A, Shapes, 4 & M & 3.75 & .74 & \\
& SD & .46 & & 1.25 \\
Combined 3 and 4 & M & 3.56 & & \\
\hline
\end{tabular}

choices as recognition responses in both groups. The subjects in Group 1 repeated $90.63 \%$ of their recalls in the recognition test, and subjects in Group 3 repeated $78.13 \%$. The usual improvement in memory performance when switching from recall to recognition was not observed in Group 1, suggesting that poorer labeling performance was not primarily a problem in accessing the correct names. With all the names available, these subjects could still not improve their performance.

The last data to be examined are the shape recognition scores for Groups 2 and 4 . These data, presented in the last column of Table 1, show shape recognition for both groups to be above the chance level of responding of 4 . The difference in the means from chance in Group 2, $\mathrm{t}(7)=3.55, \mathrm{p}<.01$, and Group 4, $t(7)=6.53, p<.001 ;$ are significant and indicate that subjects in both conditions were able to retain some of the shapes in memory. This is important as it provides a basis for attaching a verbal label. Moreover, a comparison of which shapes were recognized in Groups 2 and 4 shows that no recognition differences were obtained between shapes presented only in the critical sequence and those seen in the P-A task as well. Of the total correct in Group 2, 50\% were seen only once in the original sequence, while $50 \%$ were seen in the P-A task also. For Group 4, the figures are $49.09 \%$ and $50.91 \%$, respectively. This indicates that the subjects' retention of the shapes was not a function of exposure in the P-A task, and that subjects were able to retain some of the shapes in memory when they were only seen once for $15 \mathrm{sec}$.

More important to the difference between pre- and postexposure labeling is the finding that shape recogintion for Group 4 was better than for Group 2, even though both were significantly greater than chance. This is indicated by a comparison of the means of Groups 2 and 4, which found the differences to be significant by a two-tailed test, $\mathrm{t}(14)=2.41, \mathrm{p}<.05$.
This difference could reflect decay of the memory traces for the shapes with time, as Group 2 had a longer time span between original shape presentation and subsequent testing than Group 4 due to the interposition of the P-A task, and/or it may reflect interference by virture of viewing additional shapes in the P-A task after the critical sequence. In either event, the significant difference implies that subjects who viewed the shape sequence prior to the P-A task had less information in memory about those shapes than subjects who viewed the shapes after the P-A task. This suggests that the difference between pre- and postexposure labeling may be a function of the amount of information retained in memory, and that, with equivalent retention, the difference would disappear. It may be argued that the shapes may have been better differentiated in Groups 3 and 4 than in 1 and 2 . This would imply that the groups did not differ in terms of forgetting, but rather in terms of initial attention to detail. However, the previously cited data showing equivalent recognition for shapes with names and shapes without names is consistent with earlier work using nonsense names and shapes (Vanderplas \& Garvin, 1959) and indicates that better differentiation may not account for the results.

\section{DISCUSSION}

The results of the present experiment suggest that although forgetting does seem to occur, subjects are able to label their earlier nonverbal experiences. As such, it is appropriate to ask how the problem of poor recall of early experiences may now be explained. The hypothesis offered is that the recall deficit may reflect true forgetting rather than information inaccessibility. Much of the literature on adults suggests that forgetting does not occur. Information may be temporarily inaccessible, but it is available (Tulving \& Thomson, 1973). The adult, however, has built a permanent memory structure over his lifetime to which new experiences are continually assimilated. The child, by comparison, lacks such a structure at the outset and thus may not be able to relate information and organize it as seems essential for long-term retention. Events related to the memory structure are retained, while those unrelated or perceived prior to the construction of the memory structure are permanently lost. Thus, the relationship between the appearance of language and the ability to remember early events may not be as direct as previously thought.

\section{REFERENCES}

Bower, G. H. Analysis of a mnemonic device. American Scientist, 1970, 58, 496-510.

Bruner, J. S. On cognitive growth: II. In J. S. Bruner, R. R. Olver, and P. M. Greenfield, et al (Eds.), Studies in cognitive growth. New York: Wiley, 1966, Pp. 30-67.

Gazzaniga, M. S. One brain-two minds? American Scientist, $1972,60,311-317$.

Neisser, U. Cultural and cognitive discontinuity. In T. E. Gladwin and W. Sturtevant (Eds.), Anthropology and human behavior. Washington, D. C:Anthropological Society, 1962.

Tulving, E. \& Thomson, D. M. Encoding specificity and retrieval processes in episodic memory. Psychological Review, 1973, 80, 352-373.

Vanderplas, J. M. \& Garvin, E. A. Complexity, association value, and practice as factors in shape recognition. Journal of Experimental Psychology, 1959, 57, 155-163.

(Received for publication November 25, 1974.) 